\title{
Possible indication for non-zero neutrino mass and additional neutrino species from cosmological observations
}

\author{
R. A. Burenin*1 \\ ${ }^{1}$ Space Research Institute of RAS (IKI), Moscow \\ Received December 21, 2012
}

\begin{abstract}
The constraints on total neutrino mass and effective number of neutrino species based on CMB anisotropy power spectrum, Hubble constant, baryon acoustic oscillations and galaxy cluster mass function data are presented. It is shown that the discrepancies between various cosmological data in Hubble constant and density fluctuation amplitude, measured in standard $\Lambda$ CDM cosmological model, can be eliminated if more than standard effective number of neutrino species and non-zero total neutrino mass are considered. This extension of $\Lambda$ CDM model appears to be $\approx 3 \sigma$ significant when all cosmological data are used. The model with approximately one additional neutrino type, $N_{\text {eff }} \approx 4$, and with non-zero total neutrino mass, $\Sigma m_{\nu} \approx 0.5 \mathrm{eV}$, provide the best fit to the data. In the model with only one massive neutrino the upper limits on neutrino mass are slightly relaxed. It is shown that these deviations from $\Lambda$ CDM model appear mainly due to the usage of recent data on the observations of baryon acoustic oscillations. Larger than standard number of neutrino species is measured mainly due to the comparison of the BAO data with direct measurements of Hubble constant, which was already noticed earlier. As it is shown below, the data on galaxy cluster mass function in this case give the measurement of non-zero neutrino mass.
\end{abstract}

Key words: cosmology, cosmological parameters, effective number of neutrino species, total neutrino mass

\section{INTRODUCTION}

The existence of neutrino with masses in the $\mathrm{eV}$ mass range would produce a suppression of density fluctuations on scales below the horizon when these neutrinos become non-relativistic (e.g., Hu et al., 1998). The amount of this suppression can be measured from the comparison of density fluctuations amplitude measured at early epoch from CMB anisotropy power spectrum and at recent epoch, from, e.g., galaxy cluster mass function data, which finally could give the measurement of total neutrino mass.

From cosmological observations the number of neutrino species could also be measured. Any type of neutrino, which was thermalized in early Universe, make its own contribution into the energy density of relativistic matter before equipartition. The change of this energy density result in a change in Universe expansion rate and therefore in changes of the size of sound horizon and photon diffusion scale (Silk damping scale).

All these quantities can be measured using the data from various cosmological observations. For example, the information on density fluctuation amplitude at early epoch, size of sound horizon, Silk damping scale is contained in CMB anisotropy power spectrum which

*e-mail: rodion@hea.iki.rssi.ru is now accurately measured in various experiments, such as WMAP (e.g., Larson et al.,, 2011), South Pole Telescope (Keisler et al., 2011; Storv et al., 2012), Atacama cosmology telescope (Dunklev et al., 2011), in very near future the results of CMB measurements obtained with Planck space observatory should also be published (Planck Collaboration, 2011).

The linear density fluctuations amplitude at recent epoch, which is usually described using $\sigma_{8}$ parameter, may be measured by means of various methods. One of the most accurate measurement of this quantity comes from the data on galaxy cluster mass function (see, e.g., Vikhlinin et al., 2009a b; Mantz et al., 2010a; Benson et al., 2011; Burenin. Vikhlinin, 2012).

The information on the size of sound horizon is contained also in the baryon acoustic oscillations data (BAO, see, e.g., Percival et al., 2010). In addition, the direct measurements of Hubble constant (e.g., Riess et al., 2011) should also be used in order to eliminate degeneracies between various cosmological parameters. The combination of all the data discussed above allowed to obtain constraints on total neutrino mass and number of neutrino species (e.g., Vikhlinin et al., 2009b; Mantz et al., 2010b; Keisler et al., 2011; Benson et al., 2011; Burenin, Vikhlinin, 2012).

In addition to the data used in these works, substan- 
tially improved new data on BAO observations were published recently (Blake et al., 2011; Beutler et al., 2011; Padmanabhan et al., 2012; Anderson et al., 2012). It was noticed that the distance scale measured using these data in assumption of standard $\Lambda \mathrm{CDM}$ model turns out to be in some tension with the results of direct Hubble constant measurements and that this discrepancy can be eliminated with the assumption of larger than standard effective neutrino species (Mehta et al., 2012; Anderson et al., 2012). With new additional data on Hubble constant measurements taken in account (Freedman et al., 2012), this discrepancy have increased.

As it is shown below, when these new BAO data are used, there is also a discrepancy in $\sigma_{8}$ measurements inferred from the data on CMB anisotropy power spectrum in assumption of standard $\Lambda$ CDM model and obtained using the galaxy cluster mass function data from Vikhlinin et al. (2009a, b). This discrepancy can be interpreted as a suppression of density fluctuations due to the existence of neutrinos with non-zero total mass. It is shown that the best agreement with all cosmological data discussed above is achieved in model with non-zero neutrino mass and additional neutrino species.

The cosmological parameters constraints were calculated from the simulations of Monte-Carlo Markov Chains, which were done using CosmomC software (Lewis, Bridle, 2002), version of Jan. 2012. In all Figures below the contours at $68 \%$ and $95 \%$ confidence levels are shown. All numerical values of confidence intervals are given at $68 \%$ confidence level.

\section{COSMOLOGICAL DATA \\ Galaxy clusters}

For our work the data on galaxy cluster mass function measurements were taken without any changes from Vikhlinin et al. (2009a, b). In this work a sample of 86 massive galaxy clusters with masses measured with about $10 \%$ accuracy using Chandra observations (Vikhlinin et al., 2009a) was used. Distant clusters, located at $z \approx 0.4-0.9$, were selected from $400 \mathrm{~d} \mathrm{X}$-ray galaxy cluster survey, based on ROSAT pointing data (Burenin et al., 2007). Clusters in local Universe were selected using ROSAT all sky survey (see details in Vikhlinin et al., 2009a).

Likelihood functions for this cosmological dataset are available at $\mathrm{WWW}^{1}$ (see also details in Burenin. Vikhlinin 2012). Systematic uncertainties are not included in these likelihood functions. These uncertainties are discussed in detail in Vikhlinin et al. $(2009 \mathrm{~b})$ and can be taken in account separately (Vikhlinin et al., 2009b; Burenin. Vikhlinin, 2012),

\footnotetext{
${ }^{1}$ http://hea.iki.rssi.ru/400d/cosm/
}

which is done below for all measurements where these data are used. This dataset is designated below as $C L$.

\section{CMB power spectrum}

For our work we used the data of 7 -year observations of WMAP observatory (Larson et al., 2011; Komatsu et al., 2011). Likelihoods were calculated using the software taken from archive ${ }^{2}$, version 4.1. Also we used the data at small angular scale CMB anisotropy obtained with South Pole Telescope (SPT, Keisler et al., 2011). When using these data, the contribution of Sunyaev-Zeldovich effect and also the contributions of poisson and clustered sources were taken into account according to the prescription of $\S 4.1$ in Keisler et al. (2011). These data taken together are designated below as $C M B$.

\section{Hubble constant}

In our work we used the Hubble constant measurement obtained using improved calibration of supernovae type Ia absolute magnitudes, $H_{0}=73.8 \pm$ $2.4 \mathrm{~km} \mathrm{~s}^{-1} \mathrm{Mpc}^{-1}$ (Riess et al., 2011). The error here includes both statistical and systematic uncertainties. In addition to this measurement, the results of Carnegie Hubble Project were published recently, where the new Cepheid distance scale calibration, obtained using the data of Spitzer Space Telescope, was applied to the Hubble Space Telescope Key Project data and the measurement $H_{0}=$ $74.3 \pm 1.5$ (stat.) \pm 2.1 (sys.) $\mathrm{km} \mathrm{s}^{-1} \mathrm{Mpc}^{-1}$ was obtained (Freedman et al., 2012).

As compared to Riess et al. (2011), in this work the distance to maser galaxy NGC4258 was not used. Also, this work is based on a new, independent determination of the distance to LMC, therefore, the calibration of Cepheid period-luminosity relation should be considered as independent one. For distance measurements different data were also used (see details in Riess et al. 2011 and Freedman et al. 2012). Therefore, apparently, the Hubble constant measurements presented in these two works should also be considered as independent ones.

These two measurements combined in assumption of their independence and gaussian errors give the value $H_{0}=74.1 \pm 1.8 \mathrm{~km} \mathrm{~s}^{-1} \mathrm{Mpc}^{-1}$. This measurement is designated below as $H_{0}$. We note, however, that new Hubble constant measurement from Freedman et al. (2012) do not produce strong changes in the constraints presented below. The constraints obtained using $H_{0}$ measurement from Riess et al. (2011) only are also given below for reference.

\section{Baryon acoustic oscillations}

The results of new, considerably improved measurements of baryon acoustic oscillations made using the

\footnotetext{
${ }^{2}$ http://lambda.gsfc.nasa.gov/
} 
data of large spectroscopic surveys of galaxies, published recently, are also used in our work. They include the reprocessed data of SDSS Data Release 7 (Padmanabhan et al., 2012), the data of WiggleZ (Blake et al., 2011) and $6 d F$ (Beutler et al., 2011) surveys, as well as the measurements made using the data of SDSS Data Release 9 (BOSS survey, CMASS sample, Anderson et al., 2012). All these data taken together are designated below as $B A O$.

\section{$\Lambda$ CDM MODEL}

The constraints on the mean matter density in Universe, $\Omega_{m}$, the linear density fluctuations amplitude, $\sigma_{8}$, and Hubble constant, $H_{0}$, which are obtained using various cosmological datasets in standard sixparameter $\Lambda$ CDM model (e.g., Larson et al., 2011) are shown in Fig. 1. One can see that there is a discrepancy between the direct Hubble constant measurements and the measurement obtained from BAO data in assumption of $\Lambda \mathrm{CDM}$ model (see left panel of the Figure). One can also see that new BAO data produce a discrepancy between the measurements of $\sigma_{8}$ from $C M B+B A O$ and from galaxy cluster mass function data (see right panel of Fig. 1).

The discrepancy in Hubble constant measurements appears most prominently in BAO data from SDSS DR9 (see Fig. 21 and Fig. 30 in Anderson et al. 2012). This discrepancy was noticed and was discussed in detail by Anderson et al. (2012). In this work BAO data are compared to the measurement of Hubble constant from Riess et al. (2011). When another, more recent measurement of Hubble constant from Freedman et al. (2012), is taken in account, statistical significance of this discrepancy becomes more significant. It is this discrepancy, which is shown in the left panel of Fig. 1].

\section{ADDITIONAL NEUTRINO SPECIES}

The underestimated measurement of Hubble constant can be obtained from BAO data in assumption of $\Lambda \mathrm{CDM}$ cosmological model with standard number of neutrino species, if in real Universe the extra component in relativistic energy density is present at early epoch in addition to photons and three known neutrinos. In this case the Universe expansion rate is increased during the radiation-dominated era, and the size of sound horizon is therefore decreased. If one use $\Lambda$ CDM model with standard number of neutrinos, the size of sound horizon would be overestimated, as compared to its real size. Since BAO observations give the measurement of the distance in units of sound horizon, Hubble constant, measured in this way will be underestimated. Useful discussion on related subjects can be found in Eisenstein \& White (2004) and Hou et al. (2011).
Relativistic energy density in early Universe is usually parametrized using the effective number of neutrino species, $N_{\text {eff }}$ :

$$
\rho_{r}=\left[1+\frac{7}{8}\left(\frac{4}{11}\right)^{4 / 3} N_{\mathrm{eff}}\right] \rho_{\gamma}
$$

where $\rho_{\gamma}$ - energy density of photons. The constraints on $\Omega_{m}, \sigma_{8}$ and $H_{0}$ in $\Lambda C D M$ model with free effective number of neutrino species added are shown in Fig. 2, From this Figure one can see that the discrepancies in Hubble constant measurements are eliminated in this cosmological model, as expected.

\section{ADDITIONAL NEUTRINO SPECIES AND NON-ZERO TOTAL NEUTRINO MASS}

In Fig. 2 one can also see that even if additional neutrino species are introduced into $\Lambda$ CDM model, not all the discrepancies are eliminated. From the right panel of this Figure one can see that the combined data on CMB power spectrum, BAO and Hubble constant measurements suggest significantly higher value of $\sigma_{8}$, as compared to the cluster mass function data at the same value of $\Omega_{m}$.

The measurement of $\sigma_{8}$ from $C M B+B A O+H_{0}$ data is inferred from the amplitude of $\mathrm{CMB}$ temperature fluctuation, which contain the information on density fluctuation amplitude at high redshifts, $z \approx 1000$. On the other hand, the measurement of $\sigma_{8}$ from the galaxy cluster mass function data reflects the value of this quantity at recent epoch. Therefore, this discrepancy in $\sigma_{8}$ measurements can be interpreted as a result of a suppression of density fluctuations due to non-zero total neutrino mass.

The constraints on $\Omega_{m}, \quad \sigma_{8}$ and $H_{0}$ in $\Lambda C D M+N_{\text {eff }}+\Sigma m_{\nu}$ model are shown in Fig. 3. One can see that in this case all cosmological datasets are completely consistent with each other. The consistency is achieved at the expense of the detection of larger then standard effective number of neutrino species and non-zero total neutrino mass. The constraints on total neutrino mass and effective number of neutrino species obtained with all the data combined are shown in Fig. 4 and 5.

If all considered cosmological data are used $\left(C M B+B A O+H_{0}+C L\right)$, the change of $\chi^{2}$ when two parameters, $\Sigma m_{\nu}$ and $N_{\text {eff }}$, are introduced into the cosmological model turns out to be $\Delta \chi^{2}=13.0$, which corresponds to $\approx 3.2 \sigma$ significance. In this case the following measurements of effective number of neutrino species and total neutrino mass are obtained: $N_{\text {eff }}=4.03 \pm 0.36, \Sigma m_{\nu}=0.49 \pm 0.17 \mathrm{eV}$. Here and everywhere below the systematic uncertainties of galaxy cluster mass function measurements from 

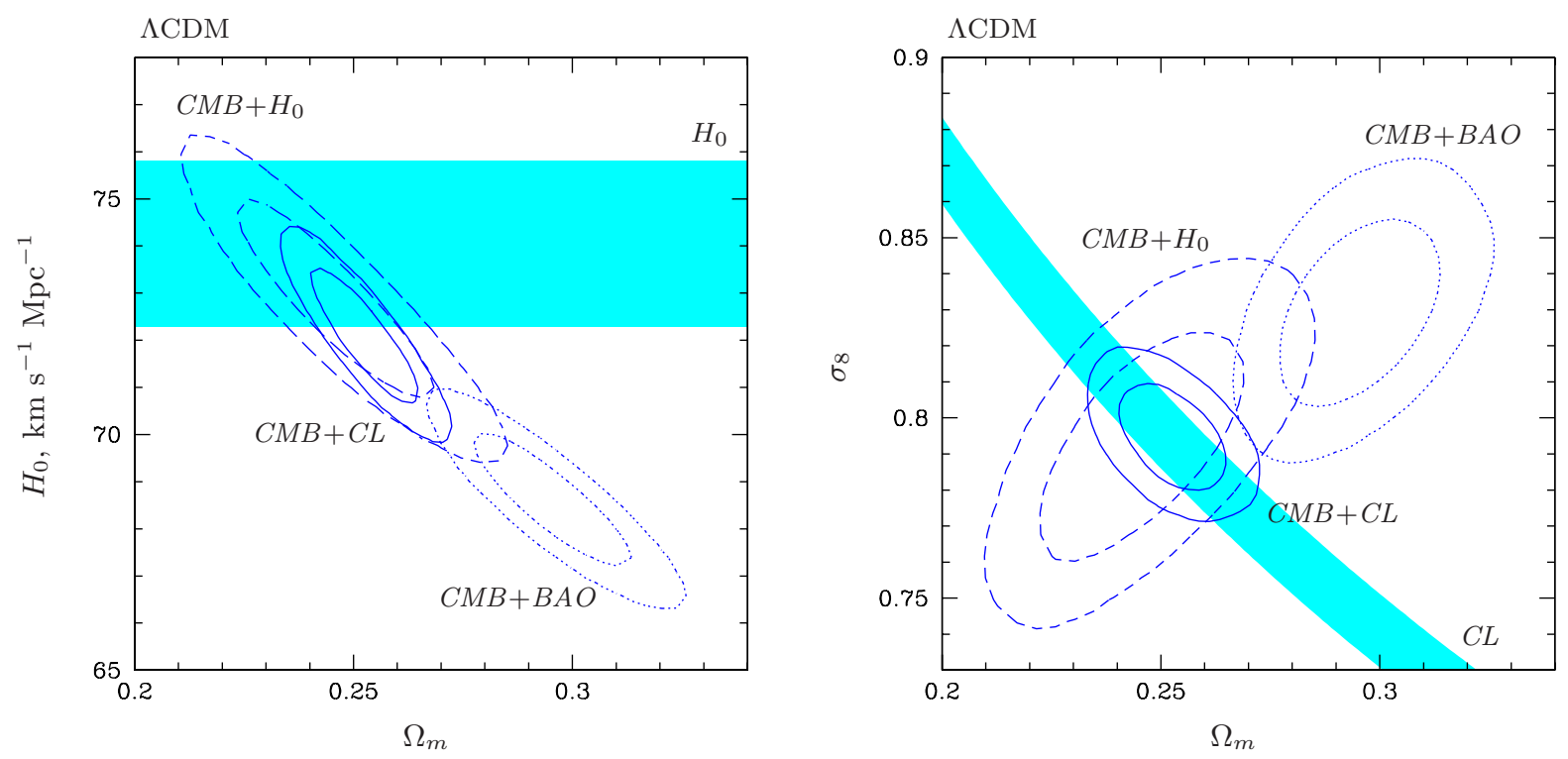

Fig. 1. The constraints on $\Omega_{m}, \sigma_{8}$ and $H_{0}$ in $\Lambda$ CDM model, obtained using various cosmological datasets. The constraints from $C M B+C L$ data are shown with solid lines, from $C M B+H_{0}$ data - with dashed lines, from $C M B+B A O$ data - with dotted lines. Shaded regions show model independent constraints (at $1 \sigma$ level) from direct measurements of Hubble constant (left) and for the combination of $\sigma_{8}$ and $\Omega_{m}$ from galaxy cluster mass function (right).
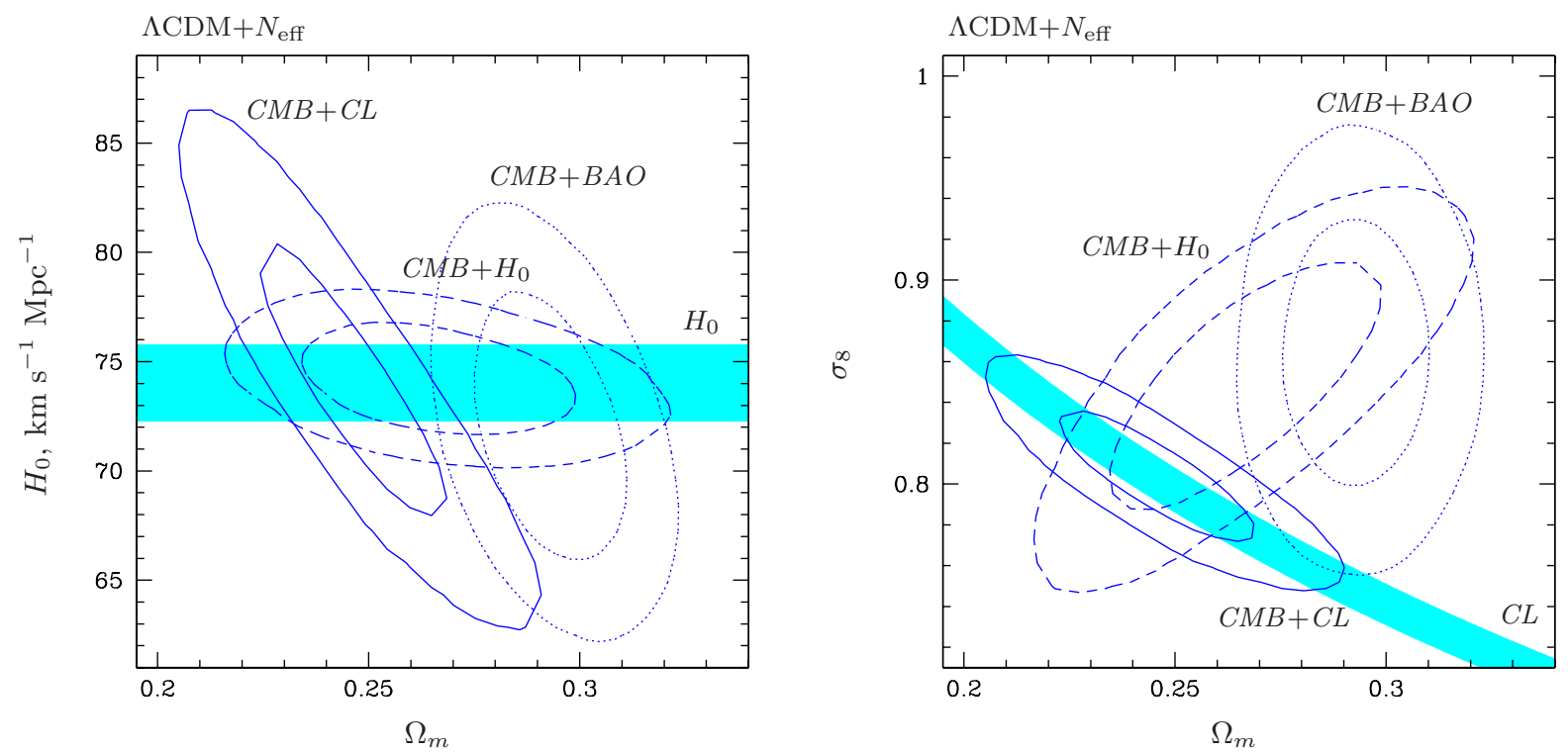

Fig. 2. The constraints on $\Omega_{m}, \sigma_{8}$ and $H_{0}$ in $\Lambda \mathrm{CDM}+N_{\text {eff }}$ model, obtained using various cosmological datasets. Contours are the same as in Fig. 1] 

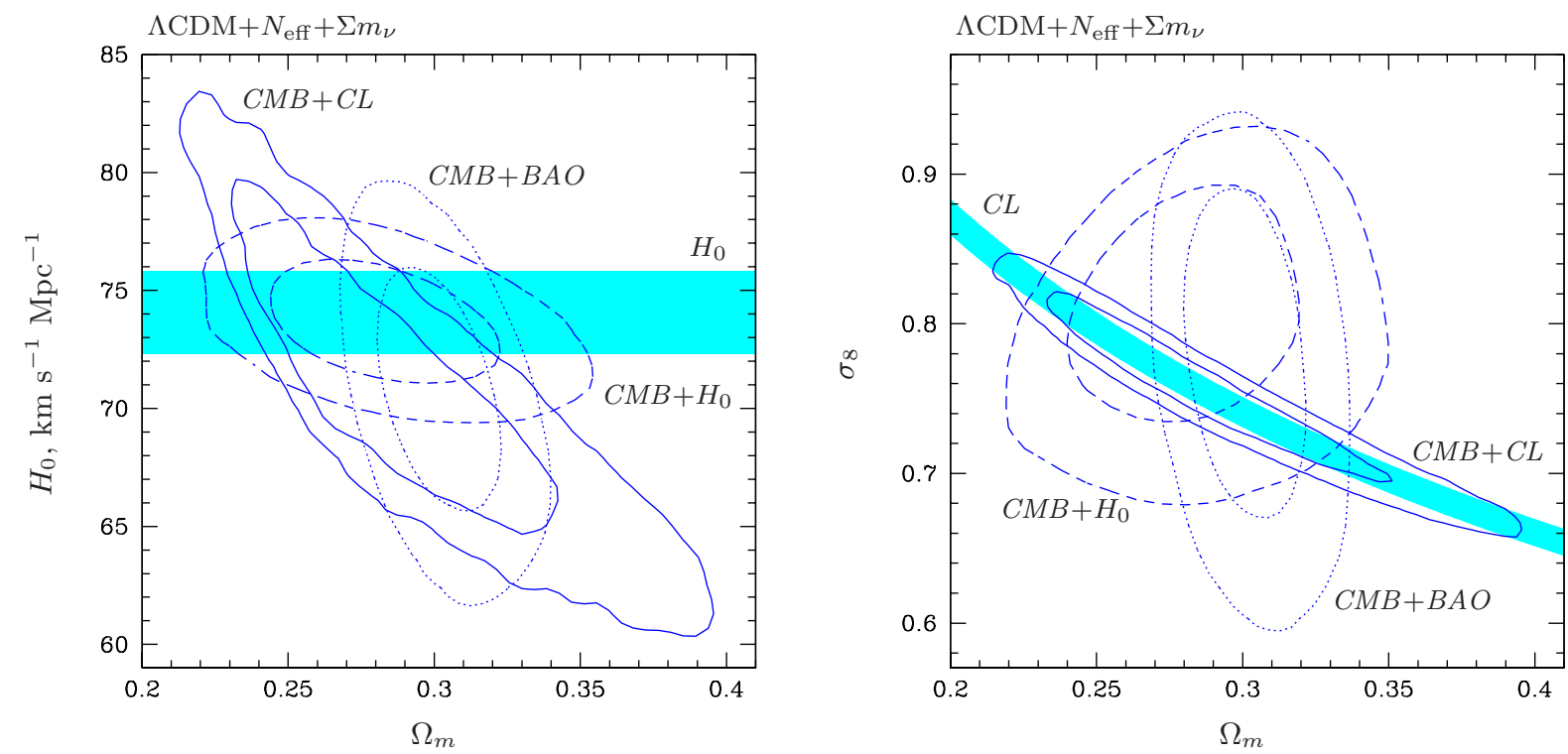

Fig. 3. The constraints on $\Omega_{m}, \sigma_{8}$ and $H_{0}$ in $\Lambda \mathrm{CDM}+N_{\mathrm{eff}}+\Sigma m_{\nu}$ model, obtained using various cosmological datasets. Contours are the same as in Fig. 11
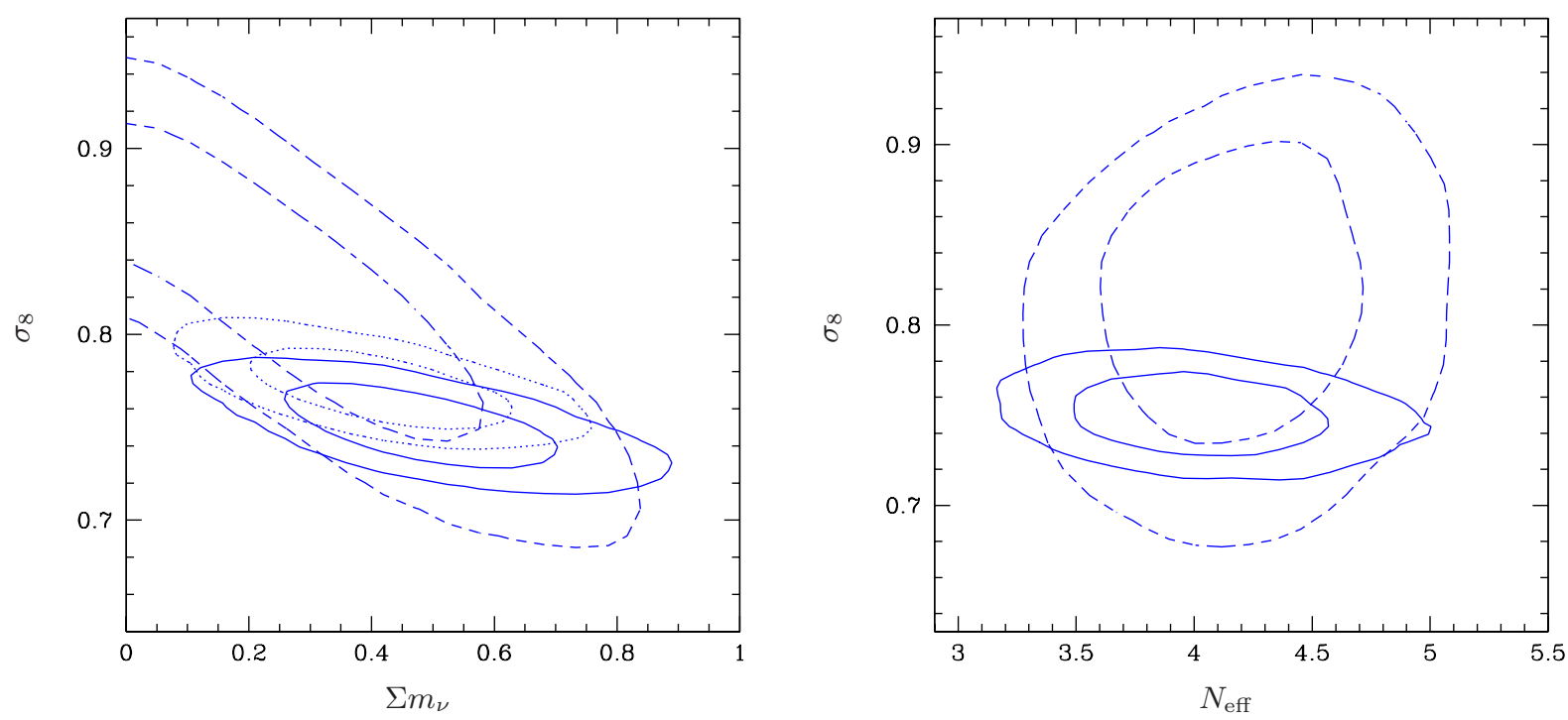

Fig. 4. The constraints on $\Sigma m_{\nu}$ and $N_{\text {eff }}$ in $\Lambda C D M+N_{\text {eff }}+\Sigma m_{\nu}$ model. The dashed contours show the constraints obtained using $C M B+B A O+H_{0}$ data, solid contours - the constraints with galaxy cluster mass function data taken in account, $C M B+B A O+H_{0}+C L$. In the left panel the contours obtained with the same data assuming that the masses of clusters in Vikhlinin et al. (2009a) are underestimated by $9 \%$ are also shown with dotted lines. 


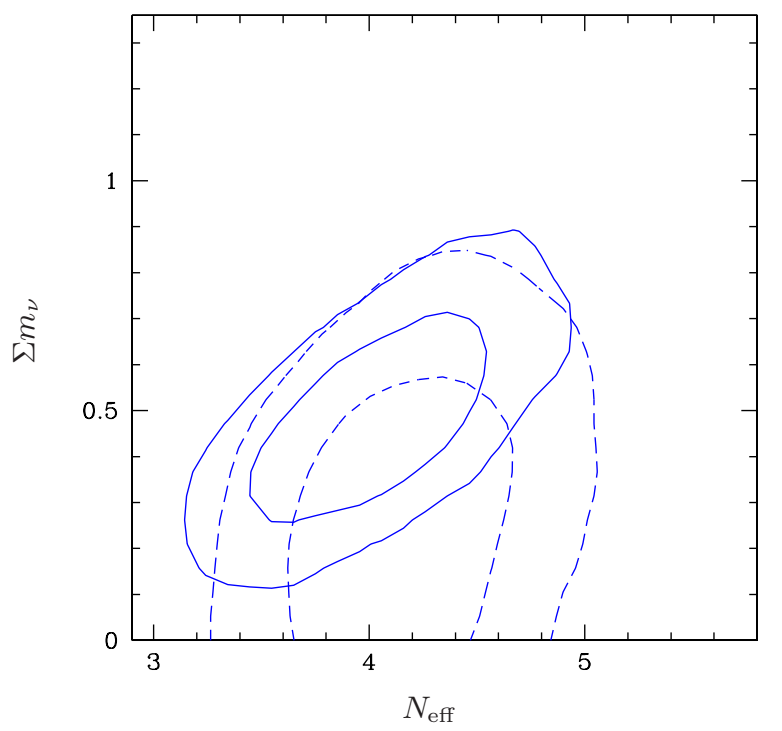

Fig. 5. The constraints on $\Sigma m_{\nu}$ and $N_{\text {eff }}$ in $\Lambda C D M+N_{\text {eff }}+\Sigma m_{\nu}$ model. The dashed contours show the constraints obtained using $C M B+B A O+H_{0}$ data, solid contours - the constraints with galaxy cluster mass function data taken in account, $C M B+B A O+H_{0}+C L$.

Vikhlinin et al. (2009b) are included in the error of total neutrino mass measurement. Note, that the measurement of effective number of neutrino species do not depend on cluster mass function data (see right panel in Fig. (4). For the case, when the measurement of Hubble constant from Riess et al. (2011) only is used, the change of $\chi^{2}$ when two parameters are added to cosmological model is $\Delta \chi^{2}=11.9$, which corresponds to $\approx 3.0 \sigma$ significance. In this case the following measurements are obtained: $N_{\text {eff }}=3.89 \pm 0.39$, $\Sigma m_{\nu}=0.47 \pm 0.16 \mathrm{eV}$.

When these constraints are obtained, the total neutrino mass is assumed to be distributed in equal parts among three neutrino types (this is the default setting in used version of CosmoMC software). Different distribution of neutrino mass can produce notable changes in total neutrino mass constraints since, with the same total neutrino mass, more massive neutrinos become non-relativistic at earlier time. Correspondent differences in total neutrino mass constraints are indeed notable when current observational data are used (e.g., Burenin. Vikhlinin, 2012). For this reason we also obtained constraints in the case when only one neutrino type is massive. These constraints are shown in Fig. 6. In this case the upper limit for total neutrino mass is somewhat relaxed, while the statistical significance of the measurement of non-zero neutrino mass remains to be approximately the same.

In this case the change of $\chi^{2}$ when two parameters are added to the model is $\Delta \chi^{2}=14.6$, which corresponds to $\approx 3.4 \sigma$ significance. In this case the follow-

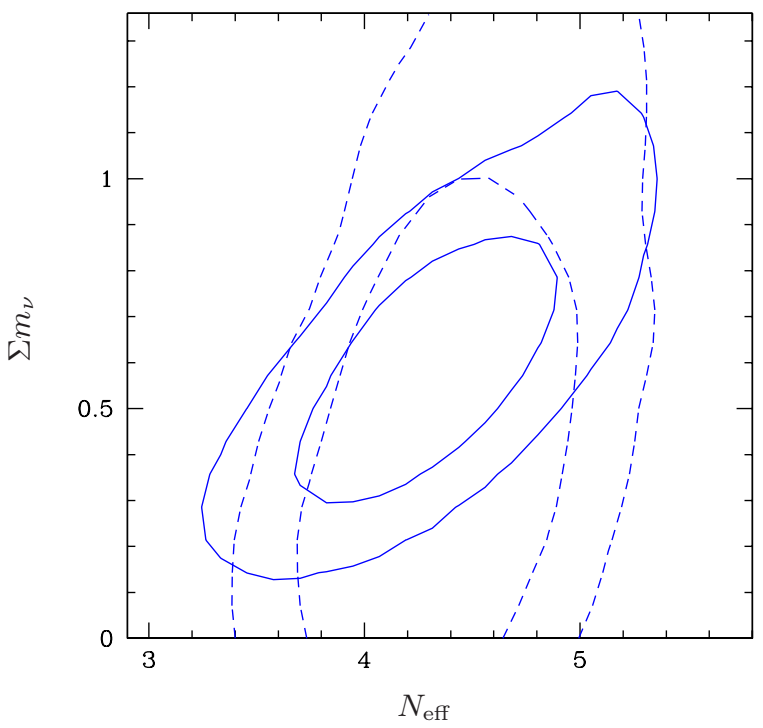

Fig. 6. The constraints on $\Sigma m_{\nu}$ and $N_{\text {eff }}$ in $\Lambda C D M+N_{\text {eff }}+\Sigma m_{\nu}$ model in the case when only one neutrino type is massive. The contours are the same as in Fig. 5

ing measurements are obtained: $N_{\text {eff }}=4.32 \pm 0.39$, $\Sigma m_{\nu}=0.60 \pm 0.22 \mathrm{eV}$. If the Hubble constant measurement from Riess et al. 2011) only is used, we get $\Delta \chi^{2}=12.8$ which corresponds to $\approx 3.1 \sigma$ significance, and in this case the measurements: $N_{\text {eff }}=4.20 \pm 0.46$, $\Sigma m_{\nu}=0.58 \pm 0.23 \mathrm{eV}$ are obtained.

\section{DISCUSSION}

As it is shown in Fig. 1, all the discrepancies in cosmological data in $\Lambda$ CDM model with zero neutrino mass and standard number of neutrino species appear mainly due to the usage of new baryon acoustics oscillations data. In fact just these data require the introduction of additional parameters into $\Lambda$ CDM model, like non-zero total neutrino mass and larger than standard number of neutrino species. If these BAO data are excluded from the consideration, all other data remain to be consistent with standard $\Lambda$ CDM model (see Fig. 1).

We note that in addition to the data used in our work, the other BAO observations exist where the lower value of Hubble constant is obtained in $\Lambda \mathrm{CDM}$ model, as compared to direct Hubble constant measurements. For example, similar discrepancy was found in the measurement of angular diameter distance from BAO observed using photometric redshifts of luminous red galaxies (Seo et al., 2012). The data on BAO observations in the transmitted flux fraction in the Ly $\alpha$ forest of high redshift quasars obtained using SDSS DR9 data (Busca et al., 2012) published recently, also give underestimated Hubble constant 
in $\Lambda$ CDM model (see. Fig. 21 in Busca et al., 2012). Note, that these BAO data are obtained using significantly different methods, as compared to BAO measurements in large spectroscopic galaxy surveys used in our work.

As it was discussed above, this discrepancy was noticed earlier, and it was also found that it can be eliminated if extra energy density of relativistic matter is present in early Universe (Mehta et al., 2012; Anderson et al., 2012; Freedman et al., 2012). Independent indications for the possibility of larger than standard value of $N_{\text {eff }}$, were also obtained earlier using the data on CMB anisotropy at smaller angular scales (Dunklev et al. 2011; Keisler et al. 2011, see also discussion in Hamann et al. 2011; Joudaki et al. 2012; Riemer-Sørensen et al. 2013). In this case the additional radiation energy density in early Universe is observed using different physical effect, by the measurement of reduced power in CMB power spectrum damping tail due to larger photon diffusion angular scale. These measurements are degenerate with primordial helium abundance and running spectral index (see details, e.g., in Dunklev et al. 2011 and Keisler et al. 2011). The data from Keisler et al. (2011) are used in our work as well, and they also have some influence on our $N_{\text {eff }}$ measurement, however, this influence is not dominant. As it was discussed above, the measurement of $N_{\text {eff }}$, presented in our work, is based mainly on the new data on BAO observations compared to the direct $H_{0}$ measurements.

From Fig. 10ne can also see that current data on direct Hubble constant measurements and cluster mass function are consistent with each other in standard $\Lambda \mathrm{CDM}$ model. In order to bring these data in accordance to recent $\mathrm{BAO}$ observations one need to decrease the measured value of Hubble constant by $7-10 \%$ and to simultaneously increase the value of $\sigma_{8}$ measured at the same $\Omega_{m}$ also by $7-10 \%$.

In our work we use two independent measurement of Hubble constant which are in good agreement with each other (Riess et al., 2011; Freedman et al., 2012). Each of them is accurate to approximately $3 \%$, including systematic errors. These are the data which give the measurement of larger than standard neutrino species when are compared to $\mathrm{BAO}$ observations.

The data on galaxy cluster mass function in their turn, when compared to the data on BAO observations, give the measurement of non-zero total neutrino mass. These constraints are affected by systematic errors of cluster mass function measurements. However, these data would be consistent with zero total neutrino mass only if the masses of clusters in Vikhlinin et al. (2009a) were underestimated by approximately $30 \%$. One can see this from the left panel in Fig. 4 where the dotted lines show the contours for all considered data $\left(C M B+B A O+H_{0}+C L\right)$, in case if cluster masses in Vikhlinin et al. (2009a) are underestimated by $9 \%$, the adopted systematic error for cluster mass scale calibration.

Systematic errors in determination of cluster mass scale is one of the main systematic uncertainties in cluster mass function measurements. In Vikhlinin et al. (2009b) the measurements of cluster masses were based on the temperature and mass of hot intracluster gas and were calibrated using hydrostatic mass measurements. Systematic error of cluster mass scale calibration was estimated as $\delta M / M \approx 0.09$, using the comparison of hydrostatic cluster masses with those based on weak lensing measurements, taken from Hoekstra (2007) and Zhang et al. (2008). More recent works, where the additional weak lensing data are presented, are in general agreement with approximately $10 \%$ systematic error for cluster masses measured using the data of X-ray observations (Israel et al., 2010, 2012; Mahdavi et al., 2012; Applegate et al., 2012).

\section{CONCLUSIONS}

In our work we show that the discrepancies between different cosmological datasets in the determination of Hubble constant and in measurements of density fluctuation amplitude in assumption of standard $\Lambda \mathrm{CDM}$ cosmological model, can be eliminated if the additional neutrino species and non-zero total neutrino mass are introduced into the cosmological model. In this case, the discrepancy in the determination of distance scale between baryon acoustic oscillations data and direct Hubble constant measurement is eliminated by the assumption of larger than standard effective number of neutrino species, which was already noticed earlier (see, e.g., Mehta et al., 2012; Anderson et al., 2012; Freedman et al., 2012; (Seo et al., 2012). The remaining discrepancy in $\sigma_{8}$ measurements between combined $\mathrm{CMB}, \mathrm{BAO}$ and Hubble constant data and the data on galaxy cluster mass function is eliminated by the assumption of non-zero neutrino mass.

The change of $\chi^{2}$ when two parameters, $N_{\text {eff }}$ and $\Sigma m_{\nu}$, are introduced into the cosmological model corresponds to $\approx 3 \sigma$ significance level. The model with approximately one additional neutrino type, $N_{\text {eff }} \approx 4$, and with non-zero total neutrino mass $\Sigma m_{\nu} \approx 0.5$ provide the best fit to the data. In model with only one massive type of neutrino the upper limits on neutrino mass are slightly relaxed.

We emphasize, that $\Lambda$ CDM model with standard number of neutrino species and zero neutrino mass appears to be no longer consistent with cosmological data due to the usage of the data on baryon acoustic oscillations, published recently. In future these results may be independently confirmed using significantly improved data on CMB anisotropy at small 


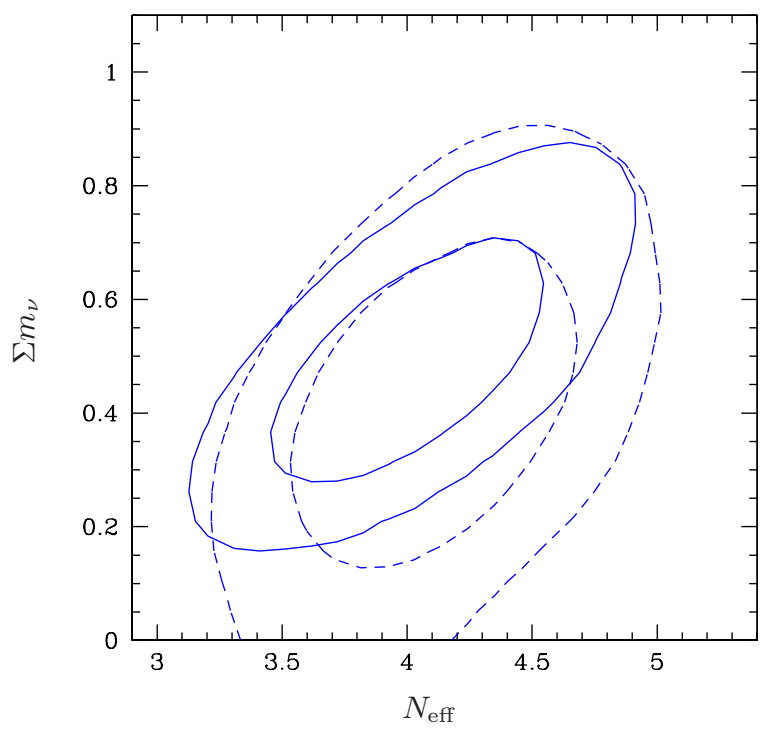

Fig. 7. The constraints on $\Sigma m_{\nu}$ and $N_{\text {eff }}$ in $\Lambda C D M+N_{\text {eff }}+\Sigma m_{\nu}$ model, obtained using WMAP9 data and also with the data on CMB lensing potential included. The dashed contours show the constraints obtained using $W M A P 9+\mathrm{SPT}+\mathrm{CMBLens}+B A O+H_{0}$ data, solid contours the constraints with galaxy cluster mass function data taken in account, $W M A P 9+\mathrm{SPT}+\mathrm{CMBLens}+\mathrm{BAO}+\mathrm{H}_{0}+C L$.

angular scales. In very near future the measurements made with Planck space observatory should be published which will probably clarify this issue.

It is known that the existence of additional light neutrino species, which could produce oscillations with $\Delta m^{2} \sim 1 \mathrm{eV}^{2}$, was suggested to explain the results of the experiments of neutrino oscillations searches at short baselines — LSDN (Aguilar et al., 2001), MiniBooNE (Aguilar-Arevalo et al., 2010), reactor antineutrino anomaly (Mueller et al., 2011; Mention et al., 2011), and gallium anomaly (Abdurashitov et al., 2006; Kaether et al., 2010; Giunti \& Laveder, 2011). In order to explain the results of these experiments the possibility of the existence of one or two light sterile neutrino was discussed (see details in, e.g., Abazajian et al., 2012).

These neutrinos can be constrained from the cosmological data as well. The constraints presented in our work are consistent with the existence of one additional type of neutrino. Since in this case $\Delta m \sim m$, the masses of all other neutrinos should be significantly lower, and to constrain these neutrinos one should use the model with only one massive type of neutrino. These constraints were obtained above and they are as follows: $N_{\text {eff }}=4.32 \pm 0.39, \Sigma m_{\nu}=0.60 \pm 0.22 \mathrm{eV}$.

\section{Note}

When this paper was already submitted for publication, few papers were published, where some new

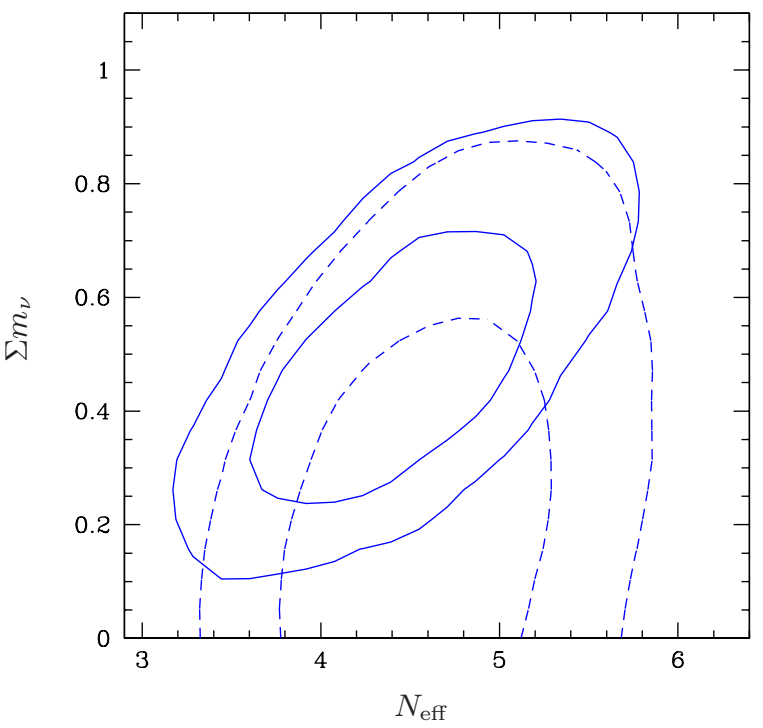

Fig. 8. The constraints on $\Sigma m_{\nu}$ and $N_{\text {eff }}$ in $\Lambda C D M+N_{\text {eff }}+\Sigma m_{\nu}$ model using all considered data, excluding the data from $S P T$. The dashed contours show the constraints obtained using $W M A P r+B A O+H_{0}$ data, solid contours - the constraints with galaxy cluster mass function data taken in account, $W M A P r+B A O+H_{0}+C L$.

constraints on number of neutrino species and total neutrino mass based on new cosmological data were presented. They are the papers on cosmological constraints obtained from nine year WMAP data (Hinshaw et al., 2012) and from new measurements of CMB anisotropy at high multipoles using the data from South Pole Telescope (SPT, Hou et al., 2012) and Atacama Cosmology Telescope (ACT, Sievers et al., 2013).

In their paper on nine year WMAP data, Hinshaw et al. (2012), obtained $N_{\text {eff }}=3.84 \pm 0.40$ in model with free $N_{\text {eff }}$ and zero total neutrino mass, in agreement with our constraints. Note, that in this work $\mathrm{CMB}$ data include also the data on power spectrum of CMB gravitational lensing potential from Das et al. (2011) and van Engelen et al. (2012), which contain the information on the density fluctuation amplitude at redshifts approximately $0.5<z<5$. If the data on density fluctuations amplitude at recent epoch are added to other current cosmological data in $\Lambda \mathrm{CDM}+N_{\text {eff }}$ model with zero neutrino mass, the lower value of $N_{\text {eff }}$ is measured due to the degeneracy between $N_{\text {eff }}$ and $\Sigma m_{\nu}$, which appears in this case Burenin, Vikhlinin 2012, see also, e.g., contours for $N_{\text {eff }}-\Sigma m_{\nu}$ in Figures above).

The effect of these new data on the constraints presented above is shown in Fig. 7 . In this Figure the constraints on number of neutrino species and total neutrino mass obtained using the latest 9year WMAP data (WMAP9, Hinshaw et al., 2012) 
and also the data on CMB lensing power spectrum amplitude (CMBLens), from Das et al. (2011) and van Engelen et al. (2012), which was taken in account as a gaussian prior, $C_{400}^{\kappa \kappa}=(3.17 \pm 0.45) \times 10^{-8}$, as it was done by Calabrese et al. (2013), are shown. The new data only slightly change the results presented above. In this case the significance of the inclusion of two parameters, $N_{\text {eff }}$ and $\Sigma m_{\nu}$ in the model is $\approx 3.3 \sigma$, and we obtained the following measurements: $N_{\text {eff }}=4.02 \pm 0.35$ and $\Sigma m_{\nu}=0.50 \pm 0.15 \mathrm{eV}$. If only one type of neutrino is massive, the correspondent significance is approximately the same and we obtain: $N_{\text {eff }}=4.12 \pm 0.36$ and $\Sigma m_{\nu}=0.58 \pm 0.21 \mathrm{eV}$. Note, that when CMB lensing power spectrum data are used, non-zero neutrino mass is obtained with lower significance even without clusters mass function data (dashed contours in Fig. 7).

The constraints based on new data from South Pole Telescope (Hou et al., 2012) in $\Lambda \mathrm{CDM}+N_{\mathrm{eff}}+\Sigma m_{\nu}$ model are generally consistent with the results, obtained in our work. From Fig. 18 of Hou et al. (2012) one can see that, similarly to our work, the measurement of larger than standard effective number of neutrino species is based mainly on the new data on BAO observations (see also the discussion in $\S 10$ of Hou et al. 2012). Also, as in our work, the measurement of non-zero neutrino mass comes from the data on galaxy clusters mass function. Therefore, as compared to our work, Hou et al. (2012) used approximately similar cosmological data and obtained approximately similar constraints on neutrino properties, as expected.

In their work, based on the new data of Atacama Cosmology Telescope $(A C T)$, Sievers et al. (2013) did not find additional neutrino types when both $\Lambda C D M+N_{\text {eff }}$ and $\Lambda C D M+N_{\text {eff }}+\Sigma m_{\nu}$ models are considered. Note, that not complete BAO dataset is used in this work (only the data from SDSS DR7, Percival et al. 2010, $6 d F$, Beutler et al. 2011, and SDSS DR9, Anderson et al. 2012). Also, as in Hinshaw et al. (2012) and Hou et al. (2012), the $H_{0}$ measurement from Freedman et al. (2012) is not used. Apparently, in the new data from $A C T$ there are some statistically insignificant discrepancies with $S P T$ data which bring the measurement of $N_{\text {eff }}$ to some lower value. However, as it was discussed above, in our work the measurements of larger than standard number of neutrinos and non-zero neutrino mass are based mainly on recent BAO data and do not depend strongly on the data on high-l CMB measurements.

In order to better show this, in Fig. 8 the constraints on $N_{\text {eff }}$ and $\Sigma m_{\nu}$ are given, which were obtained using all the data considered in our work, excluding the high- $l$ CMB data from $S P T$ (i.e., using the $W M A P r+B A O+H_{0}+C L$ dataset). There is no much change in the significance of the detection of $N_{\text {eff }}>3$ and $\Sigma m_{\nu}>0$, the main effect of the exclusion of SPT data is the relaxed upper limit for $N_{\text {eff }}$. In this case, the change of $\chi^{2}$ when two parameters, $N_{\text {eff }}$ and $\Sigma m_{\nu}$, are introduced into the model is $\Delta \chi^{2}=11.1$, which corresponds to $\approx 2.9 \sigma$ significance, and the following measurements are obtained: $N_{\text {eff }}=4.44 \pm 0.50, \Sigma m_{\nu}=0.49 \pm 0.18 \mathrm{eV}$. In the case, when only one neutrino type is massive, $\Delta \chi^{2}$ have the same value, and the following measurements are obtained: $N_{\text {eff }}=4.55 \pm 0.50, \Sigma m_{\nu}=0.54 \pm 0.24 \mathrm{eV}$.

Author is grateful to A. A. Vikhlinin for critical discussion and useful comments. In this work the results of computations made with MVS-100K supercomputer of Joint Supercomputer Center of the Russian Academy of Sciences (JSCC RAS) were used. The work is supported by Russian Foundation for Basic Research, grants 10-02-01442, 11-02-12271-ofi-m, 12-0201358, the Program for Support of Leading Scientific Schools of the Russian Federation (NSh-5603.2012.2), and the Programs of the Russian Academy of Sciences P-21/1 and OPhN-17.

\section{REFERENCES}

1. Abazajian K. N., Acero M. A., Agarwalla S. K., et al., arXiv:1204.5379.

2. Abdurashitov J.N., Gavrin V.N., Girin S.V., et al., Phys. Rev. C 73, 045805 (2006).

3. Aguilar A., Auerbach L. B., Burman R. L., et al., Phys. Rev. D 64, 112007 (2001).

4. Aguilar-Arevalo A. A., et al., MiniBooNE Collaboration, Phys. Rev. Letters 105, 181801 (2010); arXiv:1007.4730.

5. Anderson L., Aubourg E., Bailey S., et, MNRAS, in press (2012); arXiv:1203.6594.

6. Applegate D. E., von der Linden A., Kelly P. L., et al., MNRAS, in press; arXiv:1208.0605.

7. Benson B. A., de Haan T., Dudley J. P., et al., Astrophys. J., in press; arXiv:1112.5435.

8. Beutler F., Blake C., Colless M., et al., MNRAS 416, 3017 (2011).

9. Blake C., Kazin E. A., Beutler F., et al., MNRAS 418, 1707 (2011).

10. Burenin R. A., Vikhlinin A., Hornstrup A., et al., Astrophys. J. Suppl. Ser. 172, 561 (2007).

11. Burenin R. A., Vikhlinin A., Pis'ma v Astron. Zhurn. 38, 395 (2012).; [Astronomy Letters, 38, 347]

12. Busca N. G., Delubac T., Rich J., et al., Astron. Astrophys., in press, arXiv:1211.2616.

13. Calabrese E., Hlozek R.A., Battaglia N., arXiv:1302.1841.

14. Das S., Sherwin B. D., Aguirre P., et al., Phys. Rev. D 107, 021301 (2011).

15. Dunkley J., Hlozek R., Sievers J., et al., Astrophys. J. 739, 52 (2011); arXiv:1009.0866. 
16. Eisenstein D., White M., Phys. Rev. D 70, 103523 (2004).

17. Freedman W. L., Madore B. F., Scowcroft V., et al., Astrophys. J. 758, 24 (2012).

18. Giunti C., Laveder M., Phys. Rev. C 83, 065504 (2011).

19. Hamann J., Hannestad S., Raffelt G.G., Wong Y.Y.Y., J. of Cosmology and Astroparticle Phys. 09, 034 (2011).

20. Hinshaw G., Larson D., Komatsu E., et al., Astrophys. J. Suppl. Ser., in press; arXiv:1212.5226.

21. Hoekstra H., MNRAS 379, 317 (2007).

22. Hou Z., Keisler R., Knox L., Millea M., Reichardt C., Phys. Rev. D, in press;arXiv:1104.2333.

23. Hou Z., Reichardt C., Story K., et al., Astrophys. J., in press; arXiv:1212.6267.

24. Hu W., Eisenstein D. J., Tegmark M., Phys. Rev. Letters 80, 5255 (1998).

25. Israel H., Erben T., Reiprich T. H., et al., Astron. Astrophys. 520, A58 (2010).

26. Israel H., Erben T., Reiprich T. H. et al., Astron. Astrophys. 546, A79 (2012).

27. Joudaki S., Abazajian K.N., Kaplinghat M., Phys. Rev. D, in press; arXiv:1208.4354.

28. Kaether F., Hampel W, Heusser G., Kiko J., Kirsten T., Physics Lett. B 685, 47 (2010).

29. Keisler R., Reichardt C. L., Aird K. A., et al., Astrophys. J. 743, 28 (2011).

30. Komatsu E., Smith K. M., Dunkley J., Bennett C. L., Gold B., Hinshaw G., et al., Astrophys. J. Suppl. Ser. 192, 18 (2011).

31. Larson D., Dunkley J., Hinshaw G., et al., Astrophys. J. Suppl. Ser. 192, 16 (2011).

32. Lewis A., Bridle S., Phys. Rev. D 66, 103511 (2002).

33. Mahdavi A., Hoekstra H., Babul A., Astrophys. J., in press; arXiv:1210.3689.

34. Mantz A., Allen S. W., Rapetti D., Ebeling H., MNRAS 406, 1759 (2010).

35. Mantz A., Allen S. W., Rapetti D., MNRAS 406, 1805 (2010).

36. Mehta K. T., Cuesta A. J., Xu X., Eisenstein D. J., Padmanabhan N., MNRAS 427, 2168 (2012).

37. Mention G., Fechner M., Lasserre Th., et al., Phys. Rev. D 83, 073006 (2011).

38. Mueller Th. A., Lhuillier D., Fallot M., et al., Phys. Rev. C 83, 054615 (2011).

39. Padmanabhan N., Xu X., Eisenstein D. J., et al., MNRAS 427, 2132 (2012).

40. Percival W. J., Reid B. A., Eisenstein D. J., et al., MNRAS 401, 2148 (2010).

41. Planck Collaboration, Astron. Astrophys. 536, A1 (2011).

42. Riess A. G., Macri L., Casertano S., et al., Astrophys. J. 732, 129 (2011).

43. S. Riemer-Sørensen, D. Parkinson, T.M. Davis, C. Blake, Astrophys. J. 763, 89 (2013).
44. Seo H.-J., Ho S., White M., Astrophys. J. 761, 13 (2012).

45. Sievers J. L., Renee A. H., Nolta M. R., et al., J. of Cosmology and Astroparticle Phys., in press; arXiv:1301.0824.

46. Story C. L., Reichardt Z., Hou H.-J., et al., Astrophys. J., in press; arXiv:1210.7231.

47. van Engelen A., Keisler R., Zahn O., et al., Astrophys. J. 756, 142 (2012).

48. Vikhlinin A., Burenin R. A., Ebeling H., et al., Astrophys. J. 692, 1033 (2009).

49. Vikhlinin A. A., Kravtsov A. V., Burenin R. A., et al., Astrophys. J. 692, 1060 (2009).

50. Zhang Y.-Y., Finoguenov A., Böhringer H., et al., Astron. Astrophys. 482, 451 (2008). 